\title{
Exploring the Influencing Factors of Pathologic Complete Response in Estrogen Receptor-Positive Breast Cancer after Neoadjuvant Chemotherapy: A Retrospective Study
}

\section{Lingfeng Tang}

Chongqing University of Medical Science: Chongqing Medical University

\section{Xiujie Shu}

Chongqing University of Medical Science: Chongqing Medical University

Gang Tu ( $\sim$ tugang@cqmu.edu.cn)

The First Affiliated Hospital of Chongqing Medical University https://orcid.org/0000-0002-5971-4919

\section{Research Article}

Keywords: estrogen receptor-positive breast cancer, neoadjuvant chemotherapy, pathologic complete response, nomogram

Posted Date: August 23rd, 2021

DOI: https://doi.org/10.21203/rs.3.rs-806115/v1

License: (c) (i) This work is licensed under a Creative Commons Attribution 4.0 International License.

Read Full License 


\section{Abstract}

Background: The purpose of this study was to evaluate the factors associated with achieving pathologic complete response (pCR) after neoadjuvant chemotherapy (NACT) in estrogen receptor-positive(ER+) breast cancer patients, and develop a nomogram that predict the probability of achieving pCR after NACT in ER+ breast cancer patients.

Methods: A total of 431 ER+ breast cancer patients who received four cycles of thrice-weekly standard NACT and subsequently underwent surgery in the first affiliated hospital of Chongqing medical university were retrospectively enrolled, and data on their pretreatment clinicopathologic features and multiple laboratory indexes were collected. The optimal cut-off values of age, NLR, PLR and FBG were determined by the Youden index. The least absolute shrinkage and selection operator regression model was used to optimize feature selection. Forward stepwise multivariable logistic regression analysis was applied to identify predictive factors for achieving $\mathrm{PCR}$ after NACT. A nomogram was then developed according to the logistic model. Discrimination, calibration, and clinical usefulness of the predicting model were assessed using the $\mathrm{C}$-index, receiver operating characteristic curve, calibration plot, and decision curve analysis.

Results: pCR was achieved in 59 (13.7\%) patients after NACT. Multivariate analysis identified four independent factors, including tumor size, ER expression, Ki67 index and histological grade. The nomogram established based on these factors showed its discriminatory ability, with the area under the curve (AUC) of 0.774 (95\% confidence interval 0.708-0.841) and the C-index was 0.809 (95\% Cl: $0.751-$ 0.867). The calibration curve showed that the predictive ability of the nomogram was a good fit to actual observation. The decision curve showed that if the threshold is $13 \%$ and $65 \%$, using this nomogram can obtain more benefit.

Conclusions: The nomogram exhibited a sufficient discriminatory ability for predicting pCR of NACT in ER+ breast cancer patients.

\section{Introduction}

Breast cancer has become the highest incidence of cancer among women in the world. Compared with developed countries, more patients in China were diagnosed with advanced breast cancer(ABC) ${ }^{[1]}$. The primary locally advanced breast cancer( $\angle A B C)$ traditionally refers to the inoperable non-metastatic locally advanced breast cancer, including T4 tumors(the diameter $>5 \mathrm{~cm}$ or the invasion of skin and chest wall), $\mathrm{N} 2$ axillary nodes, ipsilateral supraclavicular lymph node metastasis, IBC, etc ${ }^{[2,3]}$. Neoadjuvant chemotherapy(NACT), utilized before surgery and radiotherapy, is mainly for the management of patients with ABC. By killing some proliferative and active cancer cells, NACT can effectively reduce the clinical stage of breast cancer that makes inoperable breast cancer into operable breast cancer or increases chances of breast conservation ${ }^{[4,5]}$. NACT also benefits include in vivo assessment of response to chemotherapy. A mass of studies have demonstrated that patients who achieve a pathological complete 
response (pCR) after NAC seems to obtain improved long-term outcome ${ }^{[5-7]}$. Nonetheless, Not all patients can benefit from NACT.

Based on the estrogen receptor (ER), progesterone receptor (PgR), and human epidermal growth factor receptor 2 (HER2), four main major breast cancer subtypes have been identified, including luminal $A$ and luminal B, basal-like and HER2-enriched ${ }^{[8]}$. Estrogen receptor-positive (ER+) breast cancer represents about $50 \% \sim 60 \%$, which is insensitive to chemotherapy treatment, especially in patients with Luminal A type breast cancer ${ }^{[9-13]}$. A study on NACT for stages I to III breast cancer patients at the MD Anderson Cancer Center reported that Patients with TNBC had significantly higher pCR rates compared with nonTNBC $(22 \% \text { vs } 11 \% ; \mathrm{P}=0.034)^{[11]}$. Besides Patricia $C$ et al ${ }^{[13]}$ reviewed 12 international NACT trials, including a number of 13856 patients, and found a significantly poor response to chemotherapy in the part of ER + breast cancer. The pCR rate of ER + breast cancer after NACT was less than half of that of the hormone receptor-negative breast cancer. Therefore, blindly receiving NACT in patients with ER + breast cancer may lead to worsening of the disease and may suffer from toxicities and adverse reactions associated with chemotherapy. There is a need for reliable predictors of chemosensitivity in ER + breast cancer patients that can enable screen those who can benefit from NACT.

Researches have demonstrated the correlation between clinical pathological characteristics and chemotherapy efficacy ${ }^{[14]}$. For instance, the level of ER expression and the proliferation index of Ki-67 are closely linked to chemosensitivity ${ }^{[11-16]}$. Some clinical features are also related to the efficacy of chemotherapy, including tumor size, BMI, and the metastasis of axillary lymph node, etc ${ }^{[17]}$. Furthermore, tumor microenvironment, relevant to the development and metastasis of breast cancer, in which immune and inflammatory response play an important role ${ }^{[18]}$. Some evidence has suggested that pretreatment neutrophil lymphocyte ratio (NLR) and the platelet lymphocyte ratio (PLR) reflect the inflammatory response and efficacy of chemotherapy ${ }^{[19-22]}$.

An increasing number of indicators predict the response to NACT, allowing us to screen patients who can benefit from NACT from ER + breast cancer patients. Therefore, we established a nomogram based on a comprehensive synthesis of routine clinicopathologic features calculating the probability of achieving pCR from NACT in ER + breast cancer patients.

\section{Methods}

\section{Population}

We accessed the database and screened patients between May 6, 2020 and May 31, 2020. The database was reviewed to identify all patients diagnosed from the first affiliated hospital of Chongqing medical university with ER-positive invasive breast cancer between January 1, 2012 and December 31, 2019, who received neoadjuvant chemotherapy. We used the following inclusion criteria: (I) female; (II) patients without anti-tumor treatment performed before chemotherapy ; and (III) patients with complete data. The 
exclusion criteria were: (I)patients with metastatic or other primary tumors; (II) patients with infammatory breast cancer; (III)Bilateral breast cancer. Informed consent was obtained from each patient prior to treatment. All histological specimens were paraffin-embedded and evaluated by two skilled pathologist. We divided the patients into $\mathrm{PCR}$ group and non-pCR group according to whether they reached the pCR. This study was approved by the Ethics Committee of the first affiliated hospital of Chongqing medical university(No. 2020 - 202). This article does not refer to the privacy of patients, so the informed consent was exempted. All data were fully anonymized before we accessed them. Authors is not eligible for information that could identify individual participants during or after data collection.

\section{Clinicopathologic Analysis}

Data on medical history, concurrent diseases, age, menopausal status, body mass index (BMI), tumor location, histological grade, tumor size, blood supply of tumor, lymph node status, NLR, PLR, fibrinogen(FBG) in peripheral blood, hormone receptor (HR) status, HER2 status, p53 status, Ki-67 index and NACT regimens were estimated both before NACT. The cut-off value of age, NLR, PLR and FBG were evaluated by the largest Youden index ${ }^{[23]}$. Clinical assessment of the breast depends on the MRI or breast ultrasonography. RECIST criteria was used for clinical response evaluation ${ }^{[24]}$. ER, PgR, p53, HER2 and Ki67 status were evaluated by immunohistochemistry $(\mathrm{IHC})$ in the pre-treatment core biopsy specimens. Cancers with $1 \%-100 \%$ of cells positive for ER/PgR expression are considered ER-positive/PgR-positive. HER2-positive breast cancer was defined as either HER2 gene amplification (fluorescent in situ hybridization) or were scored as $3(\mathrm{IHC})^{[3]}$. Ki-67 score was defined as the percentage of total number of tumor cells (at least 1000) with nuclear staining over 10 high powered fields $(\times 40)$. Then, pCR was defined as no residual invasive cancer in the breast or axillary lymph nodes but noninvasive breast residuals allowed after NACT.

\section{Treatment}

The criteria for receiving neoadjuvant chemotherapy in $\mathrm{HR}(+)$ breast cancer patients are as follows: The local stage of the disease is relatively late, such as patients with axillary lymph node metastasis or large mass or invasion of skin and chest wall, as well as, patients have a strong desire to do breast-conserving surgery, but don't meet requirement when diagnosed.

NACT was given according to the local protocol and national guidelines. Anthracycline-based and/or taxanebased NACT regimens were used every 3 weeks:TEC (docetaxel: $75 \mathrm{mg} / \mathrm{m} 2$, epirubicin: $75 \mathrm{mg} / \mathrm{m} 2$, and cyclophosphamide: $500 \mathrm{mg} / \mathrm{m} 2$ ); EC(epirubicin: $90 \mathrm{mg} / \mathrm{m} 2$, and cyclophosphamide: $500 \mathrm{mg} / \mathrm{m} 2$ ). In cases of HER2 amplification, None of HER2 inhibitors were added into NACT regimens. After diagnosis all patients started the first cycle of NACT in a week without other management, and received four cycles of NACT regimens.

\section{statistical method}

Statistical analysis was performed using the R software( Version 4.0.2) and SPSS (Version 25.0). the best cut-off values were determined by the largest Youden index. The categorical variables were compared 
using the Chi-squared test or the Fisher exact test. The least absolute shrinkage and selection operator (LASSO) method was used to select the optimal predictive features of NACT for ER-positive breast cancer. Features with nonzero coefficients in the LASSO regression model were selected ${ }^{[25]}$. Then, multivariable logistic regression analysis was used to build a predicting model by incorporating the feature selected in the LASSO regression model. The features were considered as odds ratio (OR) having 95\% confidence interval $(\mathrm{Cl})$ and as P-value. All potential predictors were applied to develop a predicting model for the probability of $\mathrm{pCR}$ after NACT in ER + breast cancer patients ${ }^{[26]}$. To quantify the discrimination performance of the nomogram, Harrell's C-index was measured. The intolerant abilities of the model were assessed by measuring the area under the receiver-operating characteristics (ROC) curve. Calibration curves were plotted to assess the calibration of the nomogram ${ }^{[27]}$. In this case, the calibration is the agreement between the frequencies of the observed outcomes, and the probabilities predicted by the model.

\section{Results}

\section{Patients' characteristics}

A total of 431 patients with ER-positive breast cancer who received NACT we identifed and evaluated (mean age $49.57 \pm 9.72$ years [range $21-79$ years]). 324 patients $(75.2 \%)$ achieved cPR, and 59 patients (13.7\%) achieved pCR. According to the pCR after chemotherapy the patients were divided into two groups. The optimal cut-off values were 45 for age, 178.53 for PLR, 2.22 for NLR and 2.35 for fibrinogen level. The correlations between $\mathrm{PCR}$ and clinical factors are displayed in Table 1. pCR was significantly associated with tumor size, histological grade, ER, PgR, Ki-67, and HER2 status $(p<0.05)$. 
Table 1

Baseline clinicopathological characteristics of patients with ER + breast cancer $(n=431)$.

Characteristic

$n(\%)$

Overall $(n=431) \quad \operatorname{pCR}(n=59) \quad$ Non-pCR $(n=372) \quad p$-value

\section{Clinical variable}

Age(years)

\begin{tabular}{|c|c|c|c|c|}
\hline$\bigotimes 45$ & $130(30.2)$ & $17(28.8)$ & $113(30.4)$ & 0.808 \\
\hline$\geq 45$ & $301(69.8)$ & $42(71.2)$ & $259(69.6)$ & \\
\hline \multicolumn{5}{|c|}{ BMI(kg/囚) } \\
\hline$₫ 24$ & $234(54.3)$ & $34(57.6)$ & $200(53.8)$ & 0.580 \\
\hline$\geq 24$ & 197(45.7) & $25(42.4)$ & $172(46.2)$ & \\
\hline
\end{tabular}

Tumor location

$\begin{array}{lllll}\text { Left } & 219(50.8) & 29(49.2) & 190(51.1) & 0.784 \\ \text { Right } & 212(49.2) & 30(50.8) & 182(48.9) & \end{array}$

Menopausal status

\begin{tabular}{|c|c|c|c|c|}
\hline Premenopausal & $253(58.8)$ & $40(67.8)$ & $213(57.4)$ & 0.132 \\
\hline Postmenopausal & $178(41.2)$ & 19(32.2) & $159(42.6)$ & \\
\hline \multicolumn{5}{|l|}{ PLR } \\
\hline ه178.53 & $352(81.7)$ & $44(74.6)$ & $308(82.8)$ & 0.130 \\
\hline$\geq 178.53$ & 79(18.3) & $15(25.4)$ & $64(17.2)$ & \\
\hline \multicolumn{5}{|l|}{ NLR } \\
\hline$\bowtie 2.22$ & $253(58.7)$ & $33(55.9)$ & $220(59.1)$ & 0.642 \\
\hline$\geq 2.22$ & $178(41.3)$ & $26(44.1)$ & $152(40.9)$ & \\
\hline \multicolumn{5}{|l|}{ FBG } \\
\hline$\varangle 2.35$ & $37(8.6)$ & $3(5.1)$ & $34(9.1)$ & 0.302 \\
\hline$\geq 2.35$ & 394(91.4) & $56(94.9)$ & 338(90.9) & \\
\hline
\end{tabular}

pCR, pathologic complete response; BMI, Body mass index; PLR, platelet to lymphocyte ratio; NLR, neutrophil to lymphocyte ratio; FBG, fibrinogen; ER, estrogen receptor;PgR, progesterone receptor;HER2, human epidermalgrowth factor receptor2;ER+, estrogen receptor-positive.

a $p$ values were determined by Chi square tests Bold values indicate statistical significance ( $p \otimes 0.05)$. 


\begin{tabular}{|c|c|c|c|c|}
\hline \multirow[t]{2}{*}{ Characteristic } & \multicolumn{4}{|l|}{$\mathrm{n}(\%)$} \\
\hline & Overall $(n=431)$ & $\mathrm{pCR}(\mathrm{n}=59)$ & Non-pCR(n = 372) & $p$-value ${ }^{a}$ \\
\hline \multicolumn{5}{|l|}{ Tumor size(cm) } \\
\hline$凶 2$ & $56(13.0)$ & $15(25.4)$ & $41(11.0)$ & 0.008 \\
\hline $2 \sim 5$ & $305(70.8)$ & $37(62.7)$ & $268(72.0)$ & \\
\hline$\otimes 5$ & $70(16.2)$ & $7(11.9)$ & 63(16.9) & \\
\hline \multicolumn{5}{|c|}{ Lymph node status } \\
\hline $\mathrm{cNO}$ & 195(45.3) & $28(47.5)$ & $167(45.0)$ & 0.726 \\
\hline $\mathrm{cNX}$ & $235(54.7)$ & $31(52.5)$ & $204(55.0)$ & \\
\hline \multicolumn{5}{|l|}{ Blood supply } \\
\hline Poor & $200(46.4)$ & $27(45.8)$ & $173(46.5)$ & 0.915 \\
\hline Abundant & $231(53.6)$ & $32(54.2)$ & 199(53.5) & \\
\hline \multicolumn{5}{|c|}{ Pathological variable } \\
\hline \multicolumn{5}{|c|}{ Histological grade } \\
\hline$\square$ & $24(5.6)$ & $2(3.4)$ & $22(5.9)$ & $凶 0.001$ \\
\hline$\square$ & $356(83.5)$ & $35(59.3)$ & $321(86.3)$ & \\
\hline$\square$ & $51(10.9)$ & $22(37.3)$ & $29(7.8)$ & \\
\hline \multicolumn{5}{|l|}{$\mathrm{ER}(\%)$} \\
\hline$\leq 50$ & 132(30.6) & $35(59.3)$ & $97(26.1)$ & $\llbracket 0.001$ \\
\hline$₫ 50$ & 299(69.4) & $24(40.7)$ & 275(73.9) & \\
\hline \multicolumn{5}{|l|}{$\operatorname{PgR}(\%)$} \\
\hline Negative & $124(28.8)$ & $26(44.1)$ & $98(26.4)$ & 0.001 \\
\hline $1 \sim 50$ & $155(36.0)$ & $24(40.7)$ & $131(35.2)$ & \\
\hline$₫ 50$ & 152(35.2) & $9(15.2)$ & 143(38.4) & \\
\hline Ki-67(\%) & & & & \\
\hline
\end{tabular}

pCR, pathologic complete response; BMI, Body mass index; PLR, platelet to lymphocyte ratio; NLR, neutrophil to lymphocyte ratio; FBG, fibrinogen; ER, estrogen receptor;PgR, progesterone receptor;HER2, human epidermalgrowth factor receptor2;ER+, estrogen receptor-positive.

${ }^{a} p$ values were determined by Chi square tests Bold values indicate statistical significance $(p \otimes 0.05)$. 


\begin{tabular}{|c|c|c|c|c|}
\hline \multirow[t]{2}{*}{ Characteristic } & \multicolumn{4}{|l|}{$n(\%)$} \\
\hline & Overall(n= 431) & $\operatorname{PCR}(n=59)$ & Non-pCR(n = 372) & $p$-value ${ }^{\mathrm{a}}$ \\
\hline $\mathbb{8 3 0}$ & $230(53.4)$ & $21(35.6)$ & $209(56.2)$ & 0.003 \\
\hline$\geq 30$ & $201(46.6)$ & $38(64.4)$ & $163(43.8)$ & \\
\hline \multicolumn{5}{|l|}{ HER2 status } \\
\hline Negative & $273(63.3)$ & $29(49.2)$ & $244(65.6)$ & 0.015 \\
\hline Positive & 158(36.7) & $30(50.8)$ & $128(34.4)$ & \\
\hline \multicolumn{5}{|l|}{ P53 status } \\
\hline Negative & $124(28.8)$ & $16(27.1)$ & 108(29.0) & 0.763 \\
\hline Positive & $307(71.2)$ & $43(72.9)$ & $264(71.0)$ & \\
\hline \multicolumn{5}{|c|}{ Chemotherapy regimens } \\
\hline TEC & $391(90.70)$ & $54(91.50)$ & $337(90.60)$ & 0.818 \\
\hline EC & $40(9.30)$ & $5(8.50)$ & $35(9.40)$ & \\
\hline \multicolumn{5}{|c|}{$\begin{array}{l}\text { pCR, pathologic complete response; BMI, Body mass index; PLR, platelet to lymphocyte ratio; NLR, } \\
\text { neutrophil to lymphocyte ratio; FBG, fibrinogen; ER, estrogen receptor;PgR, progesterone } \\
\text { receptor;HER2, human epidermalgrowth factor receptor2;ER+, estrogen receptor-positive. }\end{array}$} \\
\hline
\end{tabular}

\section{Feature selection}

Of clinicopathological and treatment features, 17 features were reduced to seven potential predictors on the basis of 431 patients in the cohort(Fig. 1) and were with nonzero coefficients in the LASSO regression model. These features left by screening included menopausal status, PLR index, tumor size, ER expression, $\mathrm{PgR}$ expression, $\mathrm{Ki}-67$ index and histological grade (shown in Table 2). 
Table 2

Prediction factors for Predictive factors for $\mathrm{pCR}$ in ER + breast cancer patients estimated by logistic regression analyses.

\begin{tabular}{|llllll|}
\hline Intercept and variable & \multicolumn{5}{l}{ Prediction model } \\
& $\boldsymbol{\beta}^{\mathrm{a}}$ & P-value & Odds ratio & $\mathbf{9 5 \%} \mathrm{Cl}$ & \\
\hline Intercept & -2.9909 & 0.005 & 0.050 & 0.006 & 0.399 \\
\hline Menopausal status & 0.5697 & 0.107 & 1.768 & 0.897 & 3.608 \\
\hline PLR & 0.5124 & 0.161 & 1.669 & 0.799 & 3.372 \\
\hline Histological grade & 1.3500 & $<\mathbf{0 . 0 0 1}$ & 3.857 & 1.916 & 7.814 \\
\hline Tumor size(cm) & -0.7789 & $\mathbf{0 . 0 0 7}$ & 0.459 & 0.258 & 0.801 \\
\hline ER(\%) & -0.1862 & $\mathbf{0 . 0 0 2}$ & 0.830 & 0.736 & 0.932 \\
\hline PgR(\%) & -0.2949 & 0.197 & 0.745 & 0.475 & 1.167 \\
\hline Ki-67(\%) & 0.2147 & $\mathbf{0 . 0 2 0}$ & 1.240 & 1.033 & 1.484 \\
\hline a $\beta$ is the regression coefficient. & & & & \\
\hline
\end{tabular}

\section{Development of an individualized prediction model}

The results of the multivariate logistic regression analysis among menopausal status, PLR index, tumor size, ER expression, PgR expression, Ki-67 index and histological grade are given in Table 2. In the multivariate logistic regression analysis, tumor size, ER expression, $\mathrm{Ki}-67$ index and histological grade were indicated as independent predictors for pCR of NACT in ER-positive patients. Patients with larger tumor size were less likely to achieve pCR (adjusted odds ratio [OR] $0.459,95 \%$ confidence interval [CI] $0.258-0.801, p=0.007)$. ER-positive breast patients with high histological grade had more possibility achieving a pCR (adjusted OR 3.857, 95\% Cl 1.916-7.814, $\mathrm{p}=<0.001$ ). Patients with low ER expression is more likely to achieve PCR after NACT (adjusted OR 0.830, 95\% Cl 0.736-0.932, p =0.002), while low Ki67 index indicated more difficulty to achieve PCR (adjusted OR 1.240, 95\% Cl 1.033-1.484, p = 0.020). The model that incorporated the above independent predictors was developed and presented as the nomogram(Fig. 2) According to each feature of an individual, the score can be obtained by the upper point scale. The higher total point corresponds to the higher probability of PCR after NACT.

\section{Apparent performance of the nomogram in the cohort}

The calibration curve of the nomogram for the prediction of pCR after NACT in ER-positive patients demonstrated good agreement in this cohort(Fig 3) The C-index for the prediction nomogram was 0.809 (95\% Cl: $0.751-0.867)$ for the cohort, as well as the AUC was 0.774 (95\% $\mathrm{Cl} 0.708-0.841)$, which indicated that the nomogram had quite a good discriminatory capability. The ROC curve of the 
nomogram is shown in Fig 4 . The decision curve analysis for the nomogram is presented in Fig 5 . The decision curve showed that if the threshold is $13 \%$ and $65 \%$, using this nomogram can obtain more benefit.

\section{Conclusions}

Nowadays the widespread use of NACT in the treatment of breast cancer leads to the increase of breast conserving rate, reduction of recurrence rate and the appropriate use of drugs. Furthermore, robust evidence demonstrates achieving pCR after NACT indicates a good prognosis ${ }^{[5-7,28]}$. As is known to us, $\mathrm{ER}+$ breast cancer is insensitive to chemotherapeutics, thus, ER+ breast cancer patients have the larger possibility of suffering from side effects of NACT but without efficacy. It is imperative to establish a model to predict the sensitivity of neoadjuvant chemotherapy in ER+ breast cancer patients.

In our study, age, menstrual status and BMI were not independent influencing factors of achieving $\mathrm{pCR}$ after NACT in ER+ breast cancer patients. $\mathrm{H}$ J Choi et al ${ }^{[17]}$ and $S$ Yan et al ${ }^{[29]}$ got a consistent conclusion. However, previous studies have shown that young breast cancer patients are more likely to achieve pCR after $\mathrm{NACT}^{[30]}$. Rong Guo et al thought age is an independent feature for predicting the probability of achieving an axillary pCR after NACT in patients with ER+ breast cancer ${ }^{[31]}$. The hypothesis demonstrated in premenopausal patient with high estrogen level chemotherapy can kill active tumor cells better because ER-positive breast cancer is estrogen dependent breast cancer ${ }^{[32]}$. Unfortunately, there is no reliable clinical evidence suggesting a significant correlation between menstrual status and the efficacy of NACT.

The tumor size and node status are important for the formulation of treatment strategy. Patients with large mass $(>5 \mathrm{~cm})$ or axillary lymph node metastasis have necessary to do $\mathrm{NACT}^{[2-3]}$. Hwang et al[ ${ }^{[33]}$ showed that patients with stage cN0-1 were more likely to achieve pCR than patients with stage cN2-3 $(\mathrm{OR}=2.93,95 \% \mathrm{Cl}: 1.41 \sim 6.05, \mathrm{P}=0.004)$. Nevertheless, some studies didn't support this conclusion ${ }^{[17,29]}$. Most researches demonstrated smaller tumor was more likely to achieve pCR after NACT in breast cancer patients ${ }^{[17,29-30]}$. Although primary surgery is still recommended for early breast cancer, some studies proved that NACT has many advantages in the use of early breast cancer ${ }^{[34-36]}$. Furthermore, Ring ${ }^{[37]}$ analyzed 453 patients who had a cCR after NACT and compared the prognosis of these patients with surgery and radiotherapy alone. For surgery and no surgery, respectively, there were no significant differences in disease-free survival or overall survival (5-year, $74 \%$ vs $76 \%$; 10 -year, $60 \%$ vs $70 \%, P=0.9$ ) between the two groups. The local recurrence rate of $8 \%$ in non-surgery group was higher than that in surgery group. With the development of medicine and radiotherapy, breast cancer avoiding surgery will become a reality.

In recent years, more and more studies have explored the correlation between PLR, NLR, FBG and other inflammatory indexes with NACT efficacy in breast cancer patients, but the conclusions dispute with each 
other ${ }^{[19-22]}$. In our study, we didn't find the significant predictive value of the NLR, PLR and FBG for the efficacy of NACT in ER+ breast cancer patients. The level of inflammatory cells and mediators is not stable, so it is not accurate to predict the efficacy of chemotherapy.

Nowadays the expression levels of ER, PgR, HER2 and Ki-67 have become routine pathological indicators of breast cancer. By immunohistochemistry breast cancer can be quickly classified in five categories, and preliminarily judged the degree of malignancy. The result shows that the expression of ER and Ki-67 is an important factor to predict achieving PCR after NACT in ER+ breast cancer patients. The expression level of Ki-67 reflects the ability of tumor cell proliferation, which is closely related to the sensitivity of chemotherapy. Sueta ${ }^{[38]}$ enrolled different subtypes of breast cancer patients and multivariate analysis showed that Ki-67 was an independent factor influencing the $\mathrm{pCR}$ of ER+ breast cancer patients after NACT(OR=6.24, 95\%Cl: $1.40 \sim 27.7, \mathrm{P}=0.016)$. Wang ${ }^{[12]}$ analyzed $188 \mathrm{ER}+$ patients and the overall $\mathrm{pCR}$ rate was $9.57 \%$. The results consistent with our study showed that Ki-67 expression was significantly related to the efficacy of chemotherapy. Similar with the conclusion of previous studies, ER expression was negatively correlated with $\mathrm{PCR}$ rate in ER+ breast cancer ${ }^{[29,31]}$. In addition, a meta analysis that included 20 studies on neoadjuvant endocrine therapy(NAET) for ER+ breast cancer showed that NAET has the same curative effect on ER+ breast cancer as NACT and that NAET has fewer adverse reactions ${ }^{[39]}$. It has been proved that HER2(+) breast cancer is more malignant than HER2(-) breast cancer and HER2(+) breast cancer patients have a later stage than HER2(-) when diagnosed,therefore, our study has the large percentage of HER2(+) patients, $36.7 \%[40]$. The result showed the expression of HER2 has no relation with achieving pCR after NACT. The efficacy of neoadjuvant targeted therapy has been recognized in recent. In particularly, the combination of trastuzumab and patuzumab has a good performance in the treatment of advanced breast cancer ${ }^{[41-43]}$. However, In treatment of ER(+) breast cancer anthracycline combined with taxanes is still the main neoadjuvant chemotherapy strategy. Neoadjuvant targeted therapy may be helpful to the curative effect, but in clinical it is rarely used with anthracycline when considering the cardiotoxicity.

Here we developed and validated an easy-to-use nomogram to predict the pCR of thrice weekly standard NACT in ER+ breast cancer patients. Through this nomogram we can quickly understand the sensitivity of patients to chemotherapy and patient outcomes. The nomogram indicated that ER+ breast cancer

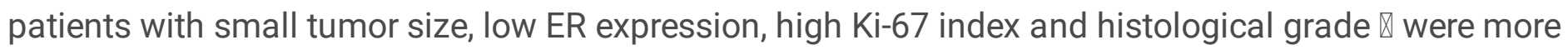
likely to achieve a pCR after NACT. With an AUC of 0.774 and a C-index of 0.809 (95\% Cl: $0.751-0.867)$, the model exhibited sufficient ability.

Although the nomogram performed a sufficient level of accuracy for predicting achieving pCR in ER+ breast cancer, this study exist several limitations. In particular, this was a retrospective study and involved a limited number of patients. The nomogram has not been validated using a large, independent, external or prospective cohort. In addition, doppler ultrasound was used to evaluate the tumor size, which has great subjectivity. Adding more sample and parameters will improve this nomogram, nevertheless, the Cindex of 0.809 suggests a sufficient level of accuracy. 
In conclusion, NACT is generally used in the treatment of breast cancer, while the patients with ER+ breast cancer who are relatively insensitive to NACT. Recognizing the outcome of the patients after NACT is an important element for determining the treatment plan. This study established a prediction model for achieving $\mathrm{pCR}$ in ER+ breast cancer patients after NACT, and screened out the patients with high sensitivity to chemotherapy. It will plays an important role in the formulation of the best treatment plan.

\section{Abbreviations}

ABC: advanced breast cancer

LABC: locally advanced breast cancer

NACT: Neoadjuvant chemotherapy

pCR: pathological complete response

ER: estrogen receptor

PgR: progesterone receptor

HER2: human epidermal growth factor receptor 2

ER+: Estrogen receptor-positive

NLR: pretreatment neutrophil lymphocyte ratio

PLR: platelet lymphocyte ratio

FBG: fibrinogen

HR: hormone receptor

IHC: immunohistochemistry

LASSO: Least absolute shrinkage and selection operator

OR: odds ratio

Cl: confidence interval

ROC: receiver-operating characteristics

\section{Declarations}

Ethics approval and consent to participate: The authors are accountable for all aspects of the work in ensuring that questions related to the accuracy or integrity of any part of the work are appropriately 
investigated and resolved. The study was conducted in accordance with the Declaration of Helsinki (as revised in 2013). The study was approved by institutional ethics board of Chongqing medical university(No. 2020-202) and individual consent for this retrospective analysis was waived.

Consent for publication: Not applicable

Availability of data and materials: The datasets generated and analysed during the current study are available from the corresponding author on reasonable request.

Competing interests: The authors declare that they have no competing interests.

Funding: This work was supported by the National Natural Science Foundation of China under Grant number [81372398].

Authors' contributions: (I) Conception and design: Gang Tu, Lingfeng Tang; (II) Administrative support: Lingfeng Tang, Xiujie Shu; (III) Provision of study materials or patients: Lingfeng Tang, Xiujie Shu; (IV) Collection and assembly of data: Lingfeng Tang, Xiujie Shu; (V) Data analysis and interpretation: Lingfeng Tang; (VI) Manuscript writing: All authors; (VII) Final approval of manuscript: All authors.

Acknowledgements:Not applicable

\section{Footnote}

Reporting Checklist: The authors have completed the CONSORT reporting checklist.

Conflicts of Interest: The authors have no conflicts of interest to declare that are relevant to the content of this article.

\section{References}

1. Fan L, Strasser-Weippl K, Li JJ, St Louis J, Finkelstein DM, Yu KD, et al. Breast cancer in China. Lancet Oncol. 2014 Jun;15(7):e279-89. https://doi.org/10.1016/S1470-2045(13)70567-9.

2. Cardoso F. Costa A, Senkus E. Aapro M, André F. Barrios CH, et al. 3rd ESO-ESMO International Consensus Guidelines for Advanced Breast Cancer (ABC 3). Ann Oncol. 2017 Dec 1;28(12):3111. https://doi.org/10.1093/annonc/mdx036. Erratum for: Ann Oncol. 2017 Jan 1;28(1):16-33..

3. National Comprehensive Cancer Network. NCCN Clinical Practice Guidelines in Oncology(NCCN Guidelines):Breast Cancer Version 2020. [2020-01-15]

https://www.nccn.org/professionals/physician_gls/pdf/breast.pdf.

4. Frei E 3rd, Miller D. Clark JR, Fallon BG. Ervin TJ. Clinical and scientific considerations in preoperative (neoadjuvant) chemotherapy. Recent Results Cancer Res. 1986;103:1-5. https://doi.org/10.1007/978-3-642-82671-9_1. 
5. van der Hage JA, van de Velde CJ, Julien JP. Tubiana-Hulin M, Vandervelden C. Duchateau L. Preoperative chemotherapy in primary operable breast cancer: results from the European Organization for Research and Treatment of Cancer trial 10902. J Clin Oncol. 2001 Nov 15;19(22):4224-37. https://doi.org/10.1200/JC0.2001.19.22.4224.

6. Fisher ER. Wang J, Bryant J. Fisher B, Mamounas E. Wolmark N. Pathobiology of preoperative chemotherapy: findings from the National Surgical Adjuvant Breast and Bowel (NSABP) protocol B18. Cancer. 2002 Aug 15;95(4):681 - 95. https://doi.org/10.1002/cncr.10741.

7. Chollet P, Amat S, Cure H, de Latour M, Le Bouedec G, Mouret-Reynier MA, et al. Prognostic significance of a complete pathological response after induction chemotherapy in operable breast cancer. Br J Cancer. 2002 Apr 8;86(7):1041-6. https://doi.org/10.1038/sj.bjc.6600210.

8. Perou CM. Sørlie T, Eisen MB. van de Rijn M, Jeffrey SS, Rees CA, et al. Molecular portraits of human breast tumours. Nature. 2000 Aug 17;406(6797):747 - 52. https://doi.org/10.1038/35021093.

9. Haque R. Ahmed SA, Inzhakova G. Shi J, Avila C. Polikoff J, et al. Impact of breast cancer subtypes and treatment on survival: an analysis spanning two decades. Cancer Epidemiol Biomarkers Prev. 2012 Oct;21(10):1848-55. https://doi.org/10.1158/1055-9965.EPI-12-0474.

10. Sivasanker M. Sistla SC, Manwar SA. Vivekanandam S. Clinical and pathologic response following taxane based neoadjuvant chemotherapy in locally advanced breast cancer patients in a tertiary care centre in India. Indian J Cancer. 2016 Apr-Jun;53(2):220-225. https://doi.org/10.4103/0019509X.197715.

11. Liedtke C. Mazouni C, Hess KR. André F, Tordai A. Mejia JA, et al. Response to neoadjuvant therapy and long-term survival in patients with triple-negative breast cancer. J Clin Oncol. 2008 Mar 10;26(8):1275-81. https://doi.org/10.1200/JC0.2007.14.4147.

12. von Minckwitz G, Kümmel S. Vogel P, Hanusch C. Eidtmann H, Hilfrich J, et al. Neoadjuvant vinorelbine-capecitabine versus docetaxel-doxorubicin-cyclophosphamide in early nonresponsive breast cancer: phase III randomized GeparTrio trial. J Natl Cancer Inst. 2008 Apr 16;100(8):542 - 51. https://doi.org/10.1093/jnci/djn085.

13. Cortazar P. Zhang L, Untch M. Mehta K, Costantino JP. Wolmark N, et al. Pathological complete response and long-term clinical benefit in breast cancer: the CTNeoBC pooled analysis. Lancet. 2014 Jul 12;384(9938):164 - 72. https://doi.org/10.1016/S0140-6736(13)62422-8.

14. Faneyte IF. Schrama JG, Peterse JL. Remijnse PL, Rodenhuis S. van de Vijver MJ. Breast cancer response to neoadjuvant chemotherapy: predictive markers and relation with outcome. Br J Cancer. 2003 Feb 10;88(3):406 - 12. https://doi.org/10.1038/sj.bjc.6600749.

15. Petrelli F. Viale G, Cabiddu M. Barni S. Prognostic value of different cut-off levels of Ki-67 in breast cancer: a systematic review and meta-analysis of 64,196 patients. Breast Cancer Res Treat. 2015 Oct;153(3):477 - 91. https:// doi.org/10.1007/s10549-015-3559-0.

16. Colleoni M. Bagnardi V, Rotmensz N. Viale G, Mastropasqua M. Veronesi P, et al. A nomogram based on the expression of $\mathrm{Ki}-67$, steroid hormone receptors status and number of chemotherapy courses 
to predict pathological complete remission after preoperative chemotherapy for breast cancer. Eur $\mathrm{J}$ Cancer. 2010 Aug;46(12):2216-24. https://doi.org/10.1016/j.ejca.2010.04.008. PMID: 20471822.

17. Choi HJ. Ryu JM, Kim I. Nam SJ, Kim SW. Yu J, et al. Nomogram for accurate prediction of breast and axillary pathologic response after neoadjuvant chemotherapy in node positive patients with breast cancer. Ann Surg Treat Res. 2019 Apr;96(4):169-176.

https://doi.org/10.4174/astr.2019.96.4.169.

18. Allinen M. Beroukhim R, Cai L. Brennan C, Lahti-Domenici J. Huang H, et al. Molecular characterization of the tumor microenvironment in breast cancer. Cancer Cell. 2004 Jul;6(1):17-32. https://doi.org/10.1016/j.ccr.2004.06.010.

19. Ulas A. Avci N, Kos T. Cubukcu E, Olmez OF. Bulut N, et al. Are neutrophil/lymphocyte ratio and platelet/lymphocyte ratio associated with prognosis in patients with HER2-positive early breast cancer receiving adjuvant trastuzumab? J BUON. 2015 May-Jun;20(3):714 - 22..

20. Asano Y. Kashiwagi S, Onoda N. Noda S, Kawajiri H. Takashima T, et al. Predictive Value of Neutrophil/Lymphocyte Ratio for Efficacy of Preoperative Chemotherapy in Triple-Negative Breast Cancer. Ann Surg Oncol. 2016 Apr;23(4):1104-10. https://doi.org/10.1245/s10434-015-4934-0.

21. Chen Y. Chen K, Xiao X. Nie Y, Qu S. Gong C, et al. Pretreatment neutrophil-to-lymphocyte ratio is correlated with response to neoadjuvant chemotherapy as an independent prognostic indicator in breast cancer patients: a retrospective study. BMC Cancer. 2016 May 19;16:320.https://doi.org/10.1186/s12885-016-2352-8.

22. Cuello-López J. Fidalgo-Zapata A, López-Agudelo L. Vásquez-Trespalacios E. Platelet-to-lymphocyte ratio as a predictive factor of complete pathologic response to neoadjuvant chemotherapy in breast cancer. PLoS One. 2018 Nov 14;13(11):e0207224. https://doi.org/10.1371/journal.pone.0207224.

23. Fluss R. Faraggi D, Reiser B. Estimation of the Youden Index and its associated cutoff point. Biom J. 2005 Aug;47(4):458 - 72. https://doi.org/10.1002/bimj.200410135.

24. Eisenhauer EA. Therasse P, Bogaerts J. Schwartz LH, Sargent D. Ford R, et al. New response evaluation criteria in solid tumours: revised RECIST guideline (version 1.1). Eur J Cancer. 2009 Jan;45(2):228 - 47. https://doi.org/10.1016/j.ejca.2008.10.026.

25. Kidd AC. McGettrick M, Tsim S. Halligan DL, Bylesjo M. Blyth KG. Survival prediction in mesothelioma using a scalable Lasso regression model: instructions for use and initial performance using clinical predictors. BMJ Open Respir Res. 2018 Jan 30;5(1):e000240. https://doi.org/10.1136/bmjresp-2017000240.

26. Balachandran VP. Gonen M, Smith JJ. DeMatteo RP. Nomograms in oncology: more than meets the eye. Lancet Oncol. 2015 Apr;16(4):e173-80. https://doi.org/10.1016/S1470-2045(14)71116-7.

27. Kramer AA. Zimmerman JE. Assessing the calibration of mortality benchmarks in critical care: The Hosmer-Lemeshow test revisited. Crit Care Med. 2007 Sep;35(9):2052-6. https://doi.org/10.1097/01.CCM.0000275267.64078.B0.

28. Del Prete S, Caraglia M. Luce A, Montella L. Galizia G, Sperlongano P, et al. Clinical and pathological factors predictive of response to neoadjuvant chemotherapy in breast cancer: A single center 
experience. Oncol Lett. 2019 Oct;18(4):3873-3879. https://doi.org/10.3892/ol.2019.10729.

29. Yan S. Wang W, Zhu B. Pan X, Wu X. Tao W. Construction of Nomograms for Predicting Pathological Complete Response and Tumor Shrinkage Size in Breast Cancer. Cancer Manag Res. 2020 Sep 10;12:8313-8323. https://doi.org/10.2147/CMAR.S270687.

30. Chou HH. Kuo WL, Yu CC. Tsai HP, Shen SC. Chu CH, et al. Impact of age on pathological complete response and locoregional recurrence in locally advanced breast cancer after neoadjuvant chemotherapy. Biomed J. 2019 Feb;42(1):66-74. https://doi.org/10.1016/j.bj.2018.10.007.

31. Guo R. Su Y, Si J. Xue J, Yang B. Zhang Q, et al. A nomogram for predicting axillary pathologic complete response in hormone receptor-positive breast cancer with cytologically proven axillary lymph node metastases. Cancer. 2020 Aug 15;126 Suppl 16:3819-3829.

https://doi.org/10.1002/cncr.32830.

32. Luo QQ. Huang JB, Wu YT. Li X, Zhao CX. Wu H, et al. Tidal chemotherapy in premenopausal patients with hormone receptor positive breast cancer. Med Hypotheses. 2017 May;102:4-7. https://doi.org/10.1016/j.mehy.2017.03.003.

33. Hwang HW. Jung H, Hyeon J. Park YH, Ahn JS. Im YH, et al. A nomogram to predict pathologic complete response ( $\mathrm{pCR}$ ) and the value of tumor-infiltrating lymphocytes (TILs) for prediction of response to neoadjuvant chemotherapy (NAC) in breast cancer patients. Breast Cancer Res Treat. 2019 Jan;173(2):255-266. https://doi.org/10.1007/s10549-018-4981-x. Epub 2018 Oct 15.

34. Zhang F. Huang M, Zhou H. Chen K, Jin J. Wu Y, et al. A Nomogram to Predict the Pathologic Complete Response of Neoadjuvant Chemotherapy in Triple-Negative Breast Cancer Based on Simple Laboratory Indicators. Ann Surg Oncol. 2019 Nov;26(12):3912-3919. https://doi.org/10.1245/s10434-019-07655-7.

35. McGale P. Dodwell D, Taylor C. Gray R; Early Breast Cancer Trialists' Collaborative Group. Neoadjuvant chemotherapy for early breast cancer. Lancet Oncol. 2018 Mar;19(3):e130. https://doi.org/10.1016/S1470-2045(18)30120-7.

36. Mieog JS. van der Hage JA, van de Velde CJ. Neoadjuvant chemotherapy for operable breast cancer. Br J Surg. 2007 Oct;94(10):1189 - 200. https://doi.org/10.1002/bjs.5894.

37. Ring A. Webb A, Ashley S. Allum WH, Ebbs S. Gui G, et al. Is surgery necessary after complete clinical remission following neoadjuvant chemotherapy for early breast cancer? J Clin Oncol. 2003 Dec 15;21(24):4540-5. https://doi.org/10.1200/JC0.2003.05.208.

38. Sueta A. Yamamoto Y, Hayashi M. Yamamoto S, Inao T. Ibusuki M, et al. Clinical significance of pretherapeutic Ki67 as a predictive parameter for response to neoadjuvant chemotherapy in breast cancer: is it equally useful across tumor subtypes? Surgery. 2014 May;155(5):927 - 35. https://doi.org/10.1016/j.surg.2014.01.009.

39. Spring L. Gupta A, Reynolds K L, Gadd, M. A., \& Bardia, A. Abstract P5-13-02: Neoadjuvant endocrine therapy for estrogen receptor (ER) positive breast cancer: Comprehensive systematic review and meta-analysis. Cancer Research, 2016, 76. https://doi.org/10.1158/1538-7445.SABCS15-P5-13-02. 
40. Slamon DJ. Clark GM, Wong SG. Levin WJ, Ullrich A. McGuire WL. Human breast cancer: correlation of relapse and survival with amplification of the HER-2/neu oncogene. Science. 1987 Jan 9;235(4785): 177 - 82. https://doi.org/10.1126/science.3798106.

41. Hurvitz SA. Martin M, Symmans WF. Jung KH, Huang CS. Thompson AM, et al. Neoadjuvant trastuzumab, pertuzumab, and chemotherapy versus trastuzumab emtansine plus pertuzumab in patients with HER2-positive breast cancer (KRISTINE): a randomised, open-label, multicentre, phase 3 trial. Lancet Oncol. 2018 Jan;19(1):115-126. https://doi.org/10.1016/S1470-2045(17)30716-7.

42. Swain SM. Ewer MS, Viale G. Delaloge S, Ferrero JM. Verrill M, et al. Pertuzumab, trastuzumab, and standard anthracycline- and taxane-based chemotherapy for the neoadjuvant treatment of patients with HER2-positive localized breast cancer (BERENICE): a phase II, open-label, multicenter, multinational cardiac safety study. Ann Oncol. 2018 Mar 1;29(3):646-653.

https://doi.org/10.1093/annonc/mdx773.

43. Debiasi M. Polanczyk CA, Ziegelmann P. Barrios C, Cao H. Dignam JJ, et al. Efficacy of Anti-HER2 Agents in Combination With Adjuvant or Neoadjuvant Chemotherapy for Early and Locally Advanced HER2-Positive Breast Cancer Patients: A Network Meta-Analysis. Front Oncol. 2018 May 22;8:156. https://doi.org/10.3389/fonc.2018.00156.

44. (In the first paragraph of the result).

\section{Figures}

a

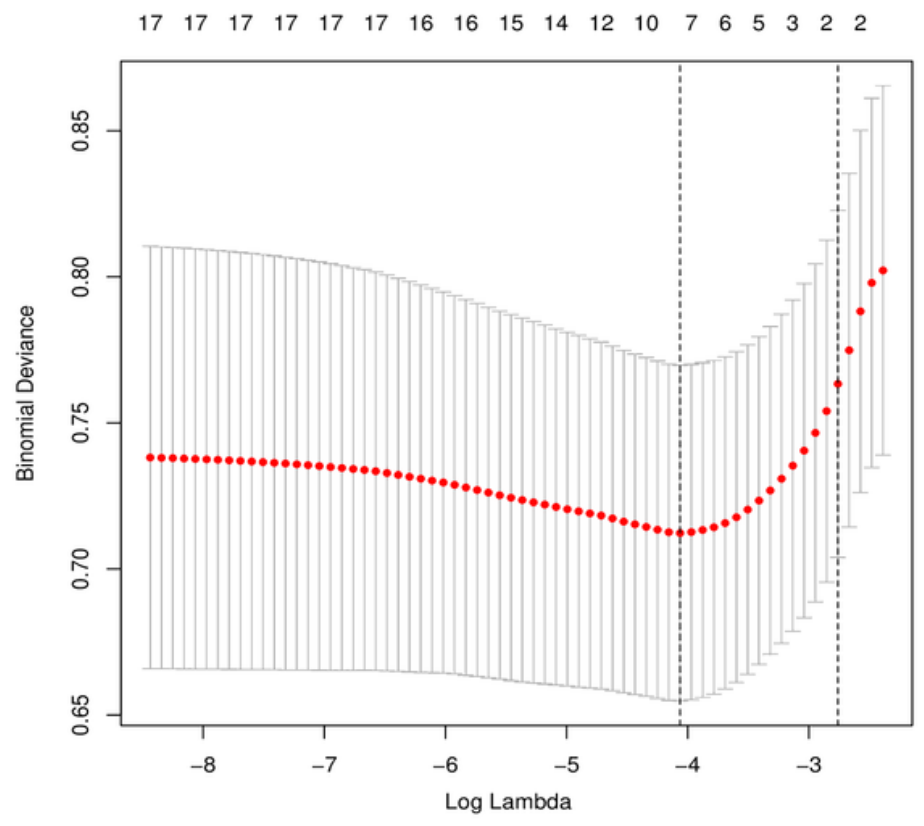

b

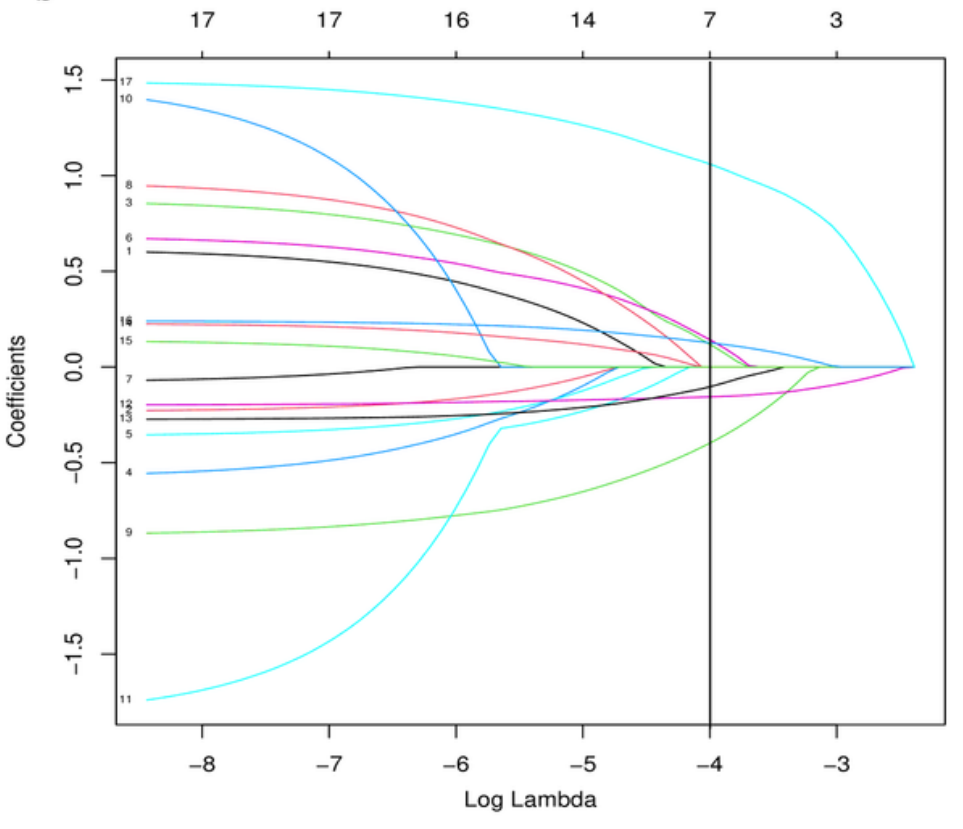

Figure 1 
Demographic and clinicopathological feature selection using the LASSO binary logistic regression model. (a) Optimal parameter (lambda) selection in the LASSO model used fivefold cross-validation via minimum criteria[25]. The partial likelihood deviance (binomial deviance) curve was plotted versus $\log (\operatorname{lambda})$. Dotted vertical lines were drawn at the optimal values by using the minimum criteria and the $1 \mathrm{SE}$ of the minimum criteria (the 1-SE criteria). (b) LASSO coefficient profiles of the 17 features. A coefficient profile plot was produced against the $\log (\operatorname{lambda})$ sequence. Vertical line was drawn at the value selected using fivefold cross-validation, where optimal lambda resulted in 7 features with nonzero coefficients. Abbreviations: LASSO, least absolute shrinkage and selection operator; SE, standard error.

Points

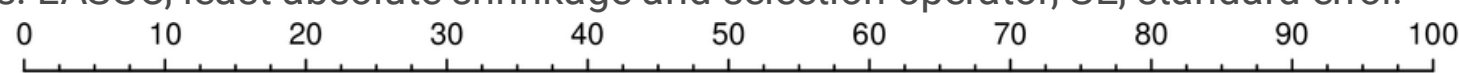

Tumor size $(\mathrm{cm})$

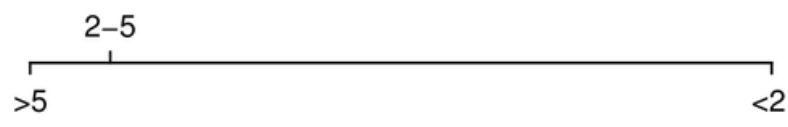

Histological grade

grade I

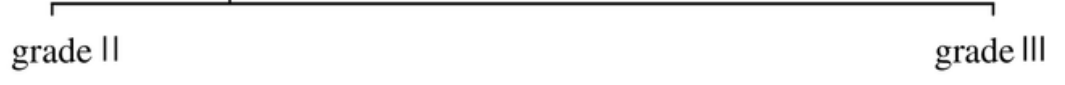

$\mathrm{ER}(\%)$

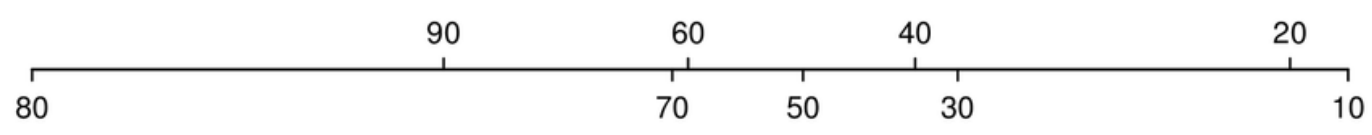

Ki67(\%)

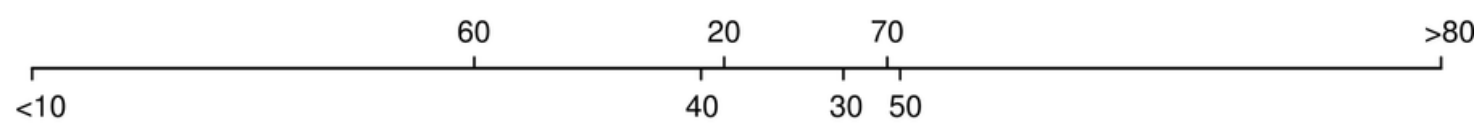

Total Points

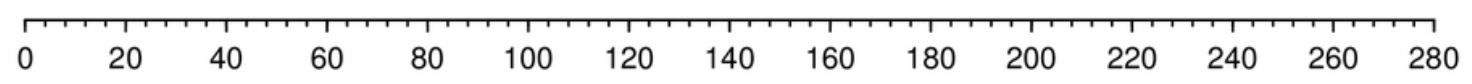

Probability of $\mathrm{pCR}$

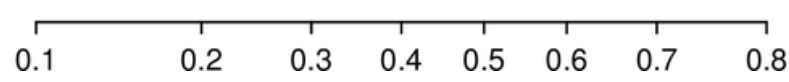

Figure 2

The nomogram the prediction of achieving pCR after NACT in ER-positive patients. The nomogram was developed in the cohort, with tumor size, ER expression, Ki-67 index and histological grade. Abbreviations: $\mathrm{pCR}$, pathologic complete response; ER, estrogen receptor; ER+, estrogen receptor-positive. 


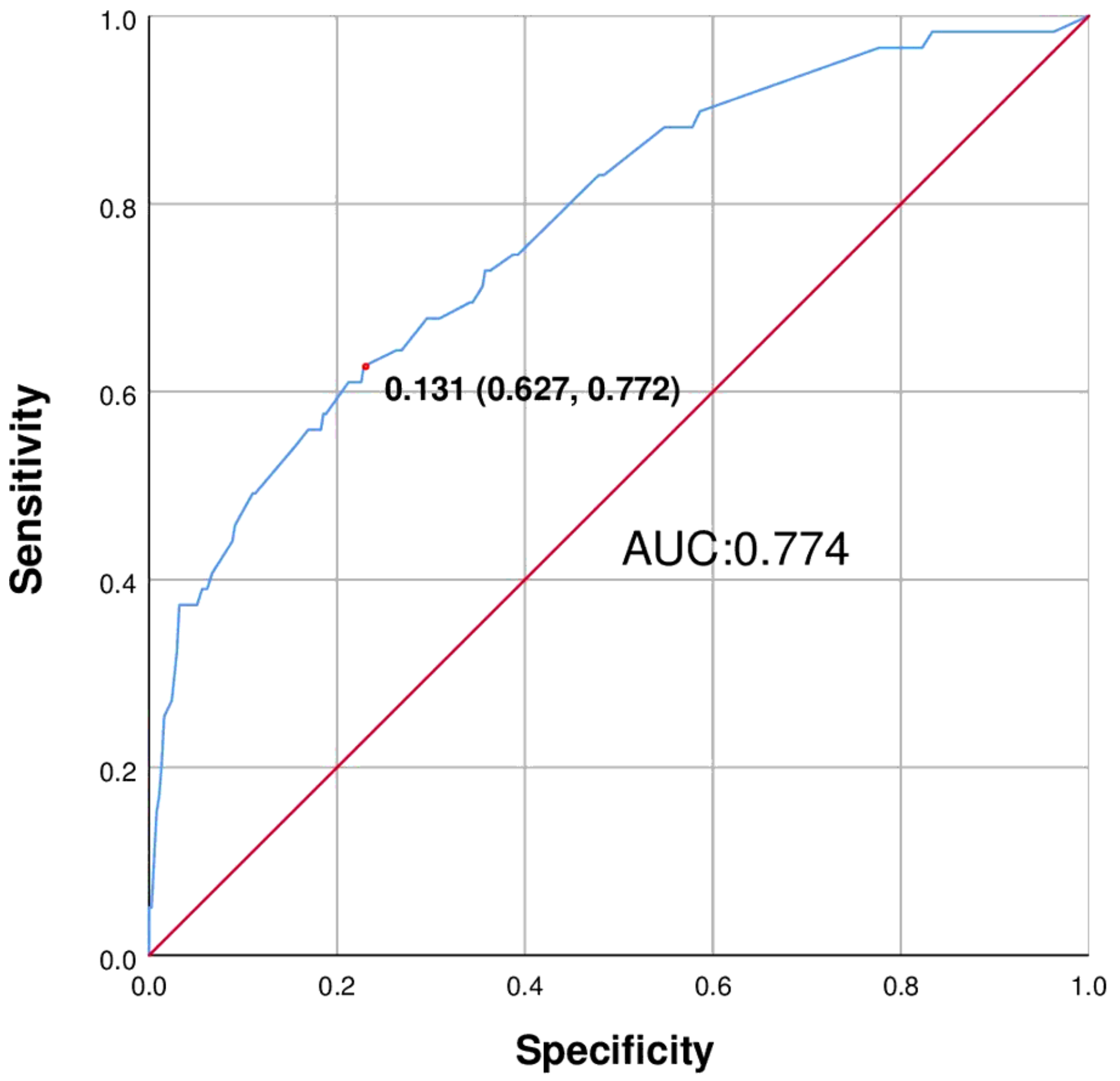

Figure 3

Receiver operating characteristic curves for the nomogram (AUC: 0.774). 


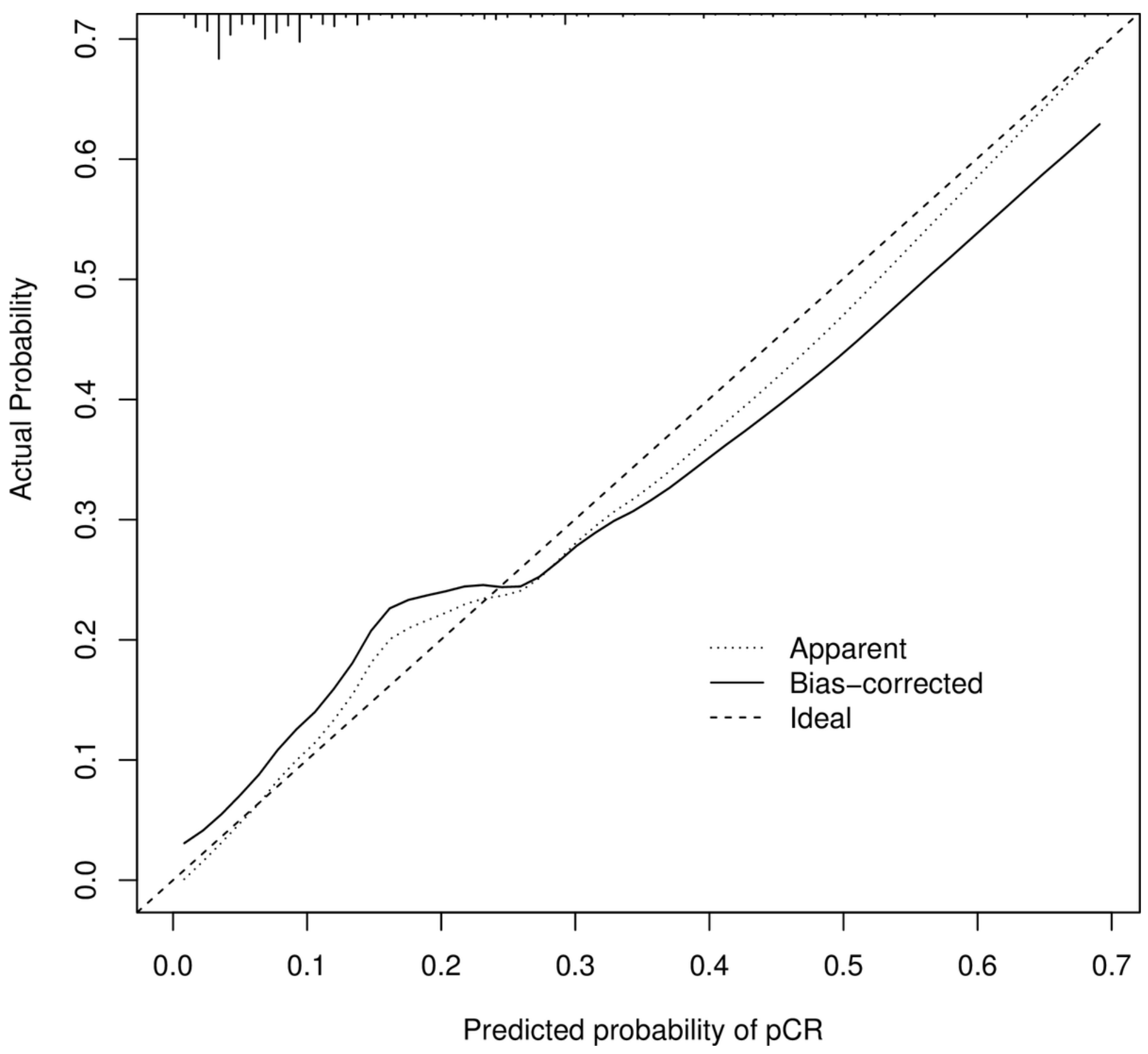

Figure 4

Calibration curves of the nomogram. The $x$-axis represents the predicted probability of pCR. The $y$-axis represents the actual probability. The diagonal dotted line represents a perfect prediction by an ideal model. The solid line represents the performance of the nomogram, of which a closer fit to the diagonal dotted line represents a better prediction. 


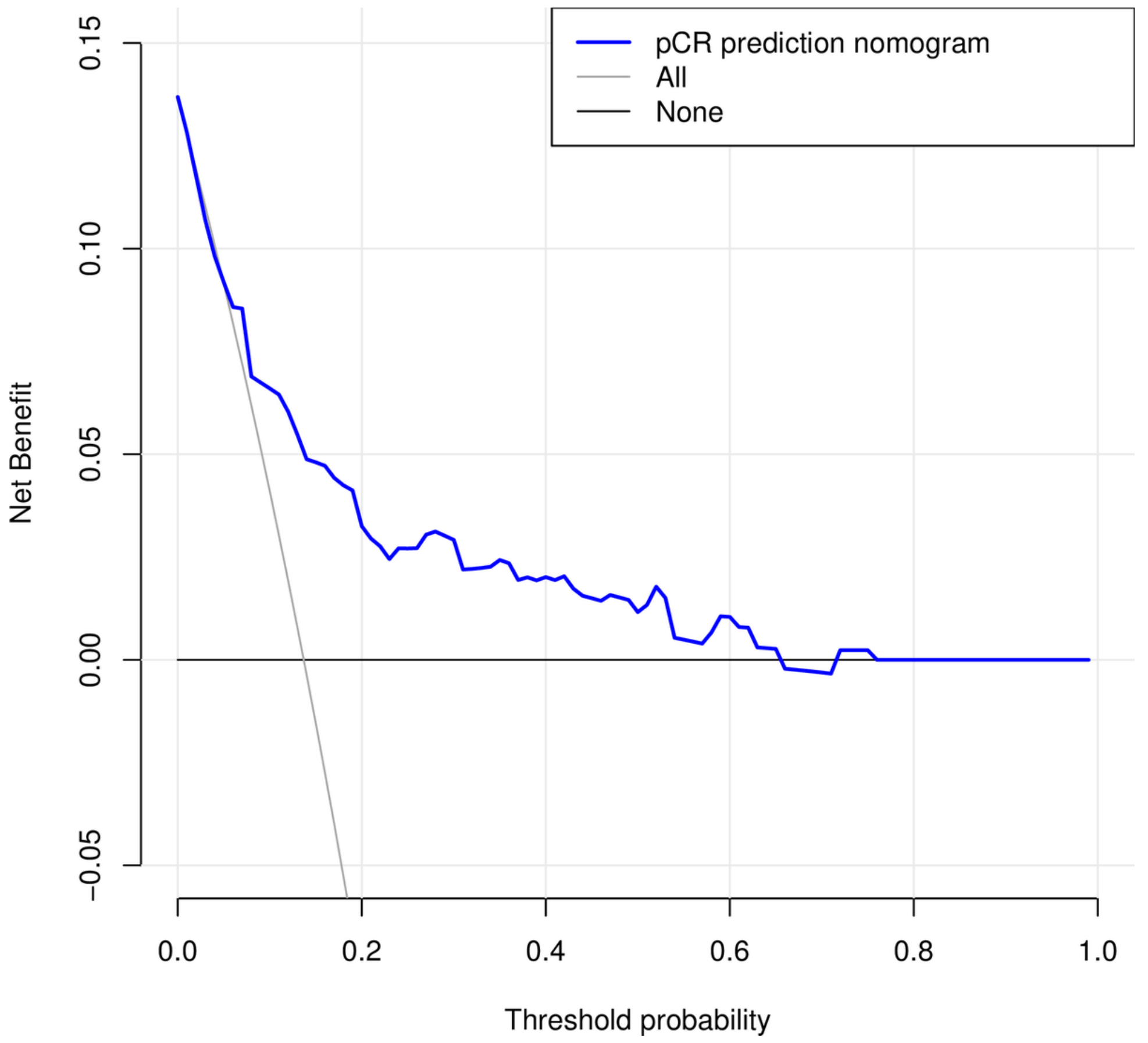

Figure 5

Decision curves for the model. The y-axis measures the net benefit. The dotted line represents the nomogram of $\mathrm{pCR}$ probability. The thin solid line represents the assumption that all patients are receiving NACT for pCR, while the parallel solid line represents the assumption that no patients are receiving NACT for $\mathrm{pCR}$. The decision curve showed that if the threshold is $13 \%$ and $65 \%$, using this nomogram can obtain more benefit. 